\title{
Ayurvedic Management of Vatarakta (gout) - A Case Report
}

\author{
*Dnyaneshwar Kantaram Jadhav \\ Shree Dhanwantari Ayurved medical college, India
}

Submission: June 15, 2017; Published: July 03, 2017

*Corresponding author: Dnyaneshwar Kantaram Jadhav, Shree Dhanwantari Ayurved medical college, Mathura, India, Tel: 9664045849; Email: dj85015@gmail.com

\section{Introduction}

In an era when modern science is progressing with advent of advanced techniques for disease, diagnosis and treatment, there is still no easy solution for innumerable chromic difficult to treat disease. Current available medicines are capable of providing pain relief and some reduction of inflammation. In old days aamvata and Sandhigata vata cases are common but Now a day's most of patients came with all joint related problems are vatarkata instead aamvata. Fast lifestyle disturbs vata and fast food dushitarakta at the same time, which gives birth to vatarakata disease. VataRakta is the disorder where in pain is predominant symptom which disturbs day-today life of the patients. Vata-rakta is an illness where both Vata and Rakta are afflicted by distinct etiological factors [1]. VataRakta is also known as-Khudaroga,Vata-balasa, Vatashra \& Adhya vata [2]. The chief complaint of the patient is severe joint pain with onset at Hasta, Pada, Mulagata sandhi [3] and then migrates to other joints in a way similar to Akhuvisha [4].

A case report as follow

A 47 year old male patient came to us with chief compliant of -

a) Dakshin gulf pradeshishul-shoth (swelling and pain at right knee joint).

b) Ubahypaadpradeshigranthiutpatii,

c) Angamarda (body ache).

d) Atisweda (excessive sweeting).

Patient had above complaints since 12 years.

No H/o Dm / HTN, Asthma

\section{History of Personal Illness}

The patient was normal 12 year back. Since then patient have been suffering from Dakshin gulf pradeshishul-shoth (swelling and pain at right knee joint), ubahypaadpradeshigranthiutpatii, [5] Angamarda (body ache), Atisweda (excessive sweeting) [6]. For this patient took treatment from different pathy doctors but got little relief in pain symptoms only, then he came to our hospital - Seth Sakharam Nemchand Jain Ayurvedic Rugnalaya in Kaychikitsa department opd. For better management we admitted patient in Ipd.

\section{Personal History}

Occupation: Work in dance bar.

Bad Habits: Chronic alcoholism.

I. Ashtavidhaparikshana

a. $\quad$ Nadi (pulse) $=78 / \mathrm{min}$.

b. Mala $($ stool $)=$ parkrut

c. Mutra (urine) $=$ Normal .

d. Jeeva $($ tounge $)=$ saam .

e. $A g n i=$ Kshudhamandya.

f. Shabda $($ speech $)=$ Normal.

g. Sparsha $($ skin $)=$ sandhipradeshishoth, ushnasparsha

h. Druka $($ eyes $)=$ Normal .

i. $\quad$ Akruti $=$ Madhyama

j. Bala= Madhyama

k. $\quad$ Raktadaaba (B.P) $=140 / 80 \mathrm{~mm} / \mathrm{Hg}$.

\section{Material and Method}

\section{Method}

a) Centre of study: S.S.N.J.AyurvedicRugnaayan, Solapur.

b) Simple random single case study.

\section{Material with Daily treatment \& prognosis: (Tables} 1-4) (Figure 1)

Clinical examination of the patients revealed regression of symptom due to our Ayurvedic management. 


\section{Global Journal of Addiction \& Rehabilitation Medicine}

Table 1: Showing matrial used in study.

\begin{tabular}{|c|c|c|c|c|}
\hline SR. NO & Dravya & Dose & Duration & Anupana \\
\hline 1. & Yograjgugul & $350 \mathrm{mg}$ & \multirow{5}{*}{$\begin{array}{c}1 \text { pack } \\
\text { BD }\end{array}$} & \multirow{5}{*}{ luck warm water } \\
\hline 2 & chopchini & $500 \mathrm{mg}$ & & \\
\hline 3 & Rasna & $500 \mathrm{mg}$ & & \\
\hline 4 & Dashamul & $500 \mathrm{mg}$ & & \\
\hline 5 & Punarnava & $500 \mathrm{mg}$ & & \\
\hline 6. & Gandhrvaharitaki & $2 \mathrm{gm}$ & 1 pack hs & luck warm water \\
\hline 7. & Kaishorgugul & $500 \mathrm{mg}$ & 1 TDS & Normal water \\
\hline 8. & Guduchighanawati & $250 \mathrm{mg}$ & 1 TDS & Normal water \\
\hline 9. & Tab. Gomutra plus & $250 \mathrm{mg}$ & $1 \mathrm{HS}$ & Normal water \\
\hline
\end{tabular}

Table 2: Showing panchkarma done in study.

\begin{tabular}{|c|}
\hline Panchkarma \\
\hline Rukshapetisweda \\
\hline
\end{tabular}

Table 3: Showing daily treatment with prognosis.

\begin{tabular}{|c|c|c|c|c|c|}
\hline Days & $1^{\mathrm{ST}}$ & $2^{\mathrm{ND}}$ & $3^{\mathrm{RD}}$ & $4^{\mathrm{TH}}$ & $5^{\mathrm{TH}}$ \\
\hline TREATMENT & $\begin{array}{l}\text { All above } \\
\text { treatment } \\
\text { as it is }\end{array}$ & $\begin{array}{l}\text { All above } \\
\text { treatment } \\
\text { as it is }\end{array}$ & $\begin{array}{c}\text { All above } \\
\text { treatment } \\
\text { as it is }+ \\
\text { virechan } \\
(1 / 4 \\
\text { Abhyadi- } \\
\text { modak } \\
+1 \text { glass } \\
\text { Triphaladi } \\
\text { Kwath ) }\end{array}$ & $\begin{array}{l}\text { All above } \\
\text { treatment } \\
\text { as it is }\end{array}$ & $\begin{array}{c}\text { All above } \\
\text { treatment } \\
\text { as it is + } \\
\text { Siravedh. } \\
\text { (nearly } 40 \\
\text { ml blood) }\end{array}$ \\
\hline \multicolumn{6}{|c|}{ Symptom } \\
\hline $\begin{array}{c}\text { gulf } \\
\text { pradesh } \\
\text { ishul }\end{array}$ & ++++ & +++ & +++ & ++ & ++ \\
\hline $\begin{array}{l}\text { gulf } \\
\text { pradesh } \\
\text { ishoth. }\end{array}$ & +++ & +++ & +++ & ++ & ++ \\
\hline $\begin{array}{l}\text { paadsandhi } \\
\text { pradesh } \\
\text { igran } \\
\text { thiutpatii }\end{array}$ & ++++ & ++++ & ++++ & +++ & +++ \\
\hline anga marda & + & ++ & ++ & + & + \\
\hline atisweda & ++ & ++ & ++ & ++ & ++ \\
\hline
\end{tabular}

\begin{tabular}{|c|c|c|c|c|c|}
\hline Days & $6^{\mathrm{TH}}$ & $7^{\mathrm{TH}}$ & $8^{\mathrm{TH}}$ & $12^{\mathrm{TH}}$ & $15^{\mathrm{TH}}$ \\
\hline TREATMENT & $\begin{array}{c}\text { All above } \\
\text { treatment } \\
\text { as it is }\end{array}$ & $\begin{array}{c}\text { All above } \\
\text { treatment } \\
\text { as it is } \\
\text { treatment } \\
\text { as it is }+ \\
\text { virechan } \\
1 / 4 \\
\text { Abhyadi- } \\
\text { modak } \\
+1 \text { glass } \\
\text { Triphaladi } \\
\text { Kwath })\end{array}$ & $\begin{array}{c}\text { All above } \\
\text { treatment } \\
\text { as it is }\end{array}$ & $\begin{array}{c}\text { All above } \\
\text { treatment } \\
\text { as it is }+ \\
\text { Siravedh. } \\
\text { (nearly } \\
40 \mathrm{ml} \\
\text { blood })\end{array}$ \\
\hline $\begin{array}{c}\text { gulf } \\
\text { pradesh } \\
\text { ishul }\end{array}$ & + & + & + & 0 & 0 \\
\hline
\end{tabular}

\begin{tabular}{|c|c|c|c|c|c|}
\hline $\begin{array}{c}\text { gulf } \\
\text { pradesh } \\
\text { ishoth. }\end{array}$ & ++ & + & 0 & 0 & 0 \\
\hline $\begin{array}{c}\text { paadsandhi } \\
\text { pradesh } \\
\text { igran } \\
\text { thiutpatii }\end{array}$ & +++ & ++ & ++ & ++ & ++ \\
\hline $\begin{array}{c}\text { anga } \\
\text { marda }\end{array}$ & + & 0 & 0 & 0 & 0 \\
\hline atisweda & + & + & + & 0 & 0 \\
\hline
\end{tabular}

Table 4: Showing changes in serum uric acid.

\begin{tabular}{|c|c|}
\hline \multicolumn{2}{|c|}{ Sr. Uric acid } \\
\hline Before (12.3.2013) & $5.14 \mathrm{mg} \%$ \\
\hline After (28.3.2013) & $3.4 \mathrm{mg} \%$ \\
\hline
\end{tabular}

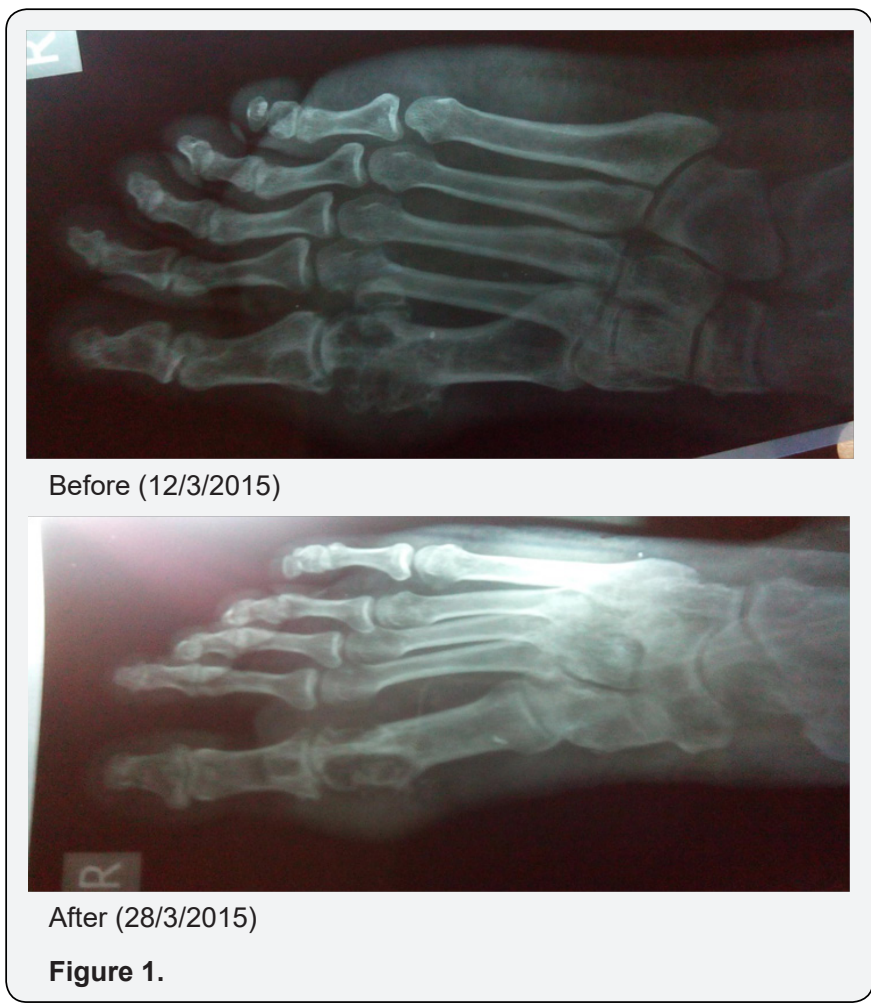

\section{Discussion}

\section{Hetu [7] of vatarkata as}

A. Ahar:

a) Improper and irregular diet causes the disturbance of vatadosha.

b) Lawan-ama-kshar food (different types of spicysaltychinize dish)

c) ShushkaAahar (whephars)

d) AmbujaanupmamsaMatsyasevan (daily)

e) Dadhi.

B. Vihar:

a) Prolong standing 
b) Ratrijagran [8-11].

C. Manasikanidan-Chinta, bhaya, and vegavrodha causesvata vrudhi.

\section{Sanprapti Ghatak}
a) Dosh : Tridosha
b) Dushya : Rasa, Rakta.
c) Adhishtana: Twaka.

\section{Action of drug \& other procedure in Management of Gout (Table 5)}

Table 5: Showing action of drug \& other procedure done in study.

\begin{tabular}{|c|c|c|}
\hline SR. NO & Dravya & Action \\
\hline 1. & Yograjgugul [8] & $\begin{array}{c}\text { Vatashulanashak, } \\
\text { strotobandhanashk }\end{array}$ \\
\hline 2 & Chopchini & aampachaka \\
\hline 3 & Rasna [9] & $\begin{array}{c}\text { Aampacahak, shothanashaka, } \\
\text { Raktaprasadak,vatashulanashak. }\end{array}$ \\
\hline 4 & Dashamul & vatashulanashak \\
\hline 5 & Punarnava [10] & Shothaghna,shaulanashaka,vatahara \\
\hline 6. & Gandhrvaharitaki & $\begin{array}{c}\text { Anuloman, vatashulanashak, } \\
\text { pittashodka }\end{array}$ \\
\hline 7. & Kaishorgugul [11] & Raktaprasadana \\
\hline 8. & Guduchighanawati & Rasayana, dhatwagni-vardhak \\
\hline 9. & Tab.Gomutra plus & Virechaka, rakatashudhikara \\
\hline 10. & Rukshapetisweda & vatashulanashak \\
\hline 11 & Virechana & Raktadosha-shodhanartha \\
\hline 12 & Siravedha & Raktadosha-shodhanartha \\
\hline
\end{tabular}

Result

The patients had started improving during hospital stay \& at end of $15^{\text {th }}$ days all symptoms nearly get disappear. As its case reached at upadrav, so its need time to cure but with Ayurved treatment its get dynamic result it relief of all subjective and objective parameter.

\section{Conclusion}

Since the therapy for vatarakata and its complication has limitation in other pathies, Ayurvedic management of chronic vatarakata can be effective therapy.

\section{References}

1. YG Joshi (2001) Charak Samhita of maharshicharak, Chakrapanidatta, commentator Charakasamhita, $5^{\text {th }}$ ed. Varanasi: Chaukambha Sanskrit sansthana; Chikitsa sthana, VatashonitaChikitsaAdhyaya 29/1; p. 627.

2. YG Joshi (2001) Charak Samhita of maharshicharak, Chakrapanidatta, commentator Charakasamhita, $5^{\text {th }}$ ed. Varanasi: Chaukambha Sanskrit sansthana; Chikitsa sthana, VatashonitaChikitsaAdhyaya 29/11; p. 656.

3. YG Joshi (2001) Charak Samhita of maharshicharak, Chakrapanidatta, commentator Charakasamhita, $5^{\text {th }}$ ed. Varanasi: Chaukambha Sanskrit sansthana; Chikitsa sthana, VatashonitaChikitsaAdhyaya 29/12; p. 657.

4. Anant Ram Sharma (2010) edited with 'susrutavimarsini' Hindi commentary. ( $1^{\text {st }}$ Ed.). Susrutasamhita,maharshisusruta. nidan-stan; Chapter 1.verse no. 48. Varanasi: Chukhambhaprakashan; page no.463.

5. YG Joshi (2001) Charak Samhita of maharshicharak, Chakrapanidatta, commentator Charakasamhita, $5^{\text {th }}$ ed. Varanasi: Chaukambha Sanskrit sansthana; Chikitsa sthana, VatashonitaChikitsaAdhyaya 29/21; p. 659.

6. YG Joshi (2001) Charak Samhita of maharshicharak, Chakrapanidatta, commentator Charakasamhita, $5^{\text {th }}$ ed. Varanasi: Chaukambha Sanskrit sansthana; Chikitsa sthana, VatashonitaChikitsaAdhyaya 29/16; p. 658.

7. YG Joshi (2001) Charak Samhita of maharshicharak, Chakrapanidatta, commentator Charakasamhita, $5^{\text {th }}$ ed. Varanasi: Chaukambha Sanskrit sansthana; Chikitsa sthana, VatashonitaChikitsaAdhyaya 29/05,06; p. 656.

8. Brahmbhanadatripahi, sharagdhar Samhita, Chaukambha Sanskrit sansthana; Varanasi: 2001.madhayma sthana; $7^{\text {th }}$ adhaya, verse no. 63, p. 203.

9. Dravyagunavidnyan part $1 \& 2$, auther professor AP Deshpande, RR Jawalgekar, Suhas Ranade, (2007) drug no.286,Anmolprakashana, pune $2,5^{\text {th }}$ edition, with reprint 2007 pg. no. 600 .

10. Dravyagunavidnyan part $1 \& 2$, auther professor AP Deshpande, RR Jawalgekar, Suhas Ranade (2007) drug no.221,Anmolprakashana, pune $2,5^{\text {th }}$ edition,with reprint pg. no. 511 .

11. Brahmbhanadatripahi, sharagdhar Samhita (2001) Chaukambha Sanskrit sansthana; Varanasi: madhayma sthana; $7^{\text {th }}$ adhaya, verse no.77, p. 205. 


\section{Your next submission with Juniper Publishers \\ will reach you the below assets}

- Quality Editorial service

- Swift Peer Review

- Reprints availability

- E-prints Service

- Manuscript Podcast for convenient understanding

- Global attainment for your research

- Manuscript accessibility in different formats ( Pdf, E-pub, Full Text, Audio)

- Unceasing customer service

Track the below URL for one-step submission https://juniperpublishers.com/online-submission.php 